\title{
Empleo de poliuretano base agua en la junta de SBR 1502/ harina natural con silanos como primers
}

\author{
Use of water-based polyurethane dispersions in adhesive \\ joint of SBR 1502/Natural flour with silanes as primers \\ Oscar Buitrago Suescún ${ }^{*} \quad$ Emilio Delgado Tobón ${ }^{1} \quad$ Mario Monroy Ramírez ${ }^{1}$ \\ Recibido 24 de agosto de 2016, aceptado 3 de julio de 2017 \\ Received: August 24, 2016 Accepted: July 3, 2017
}

\begin{abstract}
RESUMEN
Se estudió el procedimiento para lograr la adhesión de láminas compuestas con fibra natural y caucho estireno butadieno 1502, usando como adhesivo poliuretano en base agua, catalogado como ecológico. Harina de retamo liso (Teline Monspessulana), previamente sometida a tratamiento de mercerización, fue mezclada en una matriz polimérica de caucho SBR 1502, con un porcentaje de fibra de $50 \mathrm{phr}$. Se elaboraron probetas a las que se les realizaron varios tratamientos que incluyeron aplicación de 3-aminopropiltrietoxisilano y viniltrimetoxisilano como primers de adhesión, primer clorados y abrasión mecánica. Se adhirieron las probetas y fueron sometidas a ensayos de tracción al pelado para determinar cuál es el mejor procedimiento de adhesión. Estos resultados fueron analizados estructuralmente mediante caracterización con espectrometría de infrarroja por transformada de Fourier y microscopia electrónica de barrido. Los análisis permiten establecer que el silano se injertó en la superficie de fibra natural con lo que se logró la cohesión del poliuretano y el sustrato.
\end{abstract}

Palabras clave: Teline Monspessulana, SBR 1502, APTS y VTMS, adhesivo ecológico, poliuretano base agua.

\begin{abstract}
The procedure for the adherence of sheets made with 1502 styrene butadiene rubber 1502 and natural flour was studied using as water-based polyurethane dispersion which is eco-friendly. Teline monspessulana flour was previously mercerized and mixed in a polymer matrix of rubber SBR 1502 and B50\% of flour. The specimens were subjected to mechanical abrasion, after they were surface treated by via chemical with chlorine base, Vinyltrimethoxysilane and 3-(Aminopropyl) triethoxysilane primers. The adhesive joints were manufactured and then tested using the peel adhesion in order to determine the best performance. The interface between the substrates was analised by infrared spectroscopy FT-IR and scanning electron microscopy. The results indicated that the silane grafting on the surface of natural fiber achieved cohesion of the adhesive with the substrate.
\end{abstract}

Keywords: Teline Monspessulana, SBR 1502; APTES, VTMS, ecological adhesive, water-based polyurethane.

\footnotetext{
1 Programa de Ingeniería Industrial. Universidad Militar Nueva Granada. Bogotá, Colombia. E-mail: oscar.buitrago@unimilitar.edu.co; arnoldo.delgado@unimilitar.edu.co; mjmonroyr@unimilitar.edu.co

* Autor de correspondencia.
} 


\section{INTRODUCCIÓN}

Actualmente importantes industrias como la automotriz, llantera, aeronáutica y calzado utilizan como materia prima el caucho, destacándose el estireno butadieno (SBR) [1, 2]. Para este material es fundamental la adhesión caucho-caucho así como el desarrollo de nuevos materiales con carga de fibra natural $[3,4]$. El uso de fibras cortas como refuerzo en materiales de caucho proporciona estabilidad dimensional antes del curado y además contribuye en la mejora de las propiedades mecánicas del material vulcanizado [5]. Otro aporte importante está en el desarrollo de materiales que requieren una baja deformación [6,7]. Debido a las ventajas que presentan las fibras vegetales, con ellas se elaboran materiales más livianos, con baja toxicidad, sostenibles en su producción y biodegradables [8-9].

La superficie de los sustratos de materiales no poliméricos como el metal, el vidrio, fibras naturales, etc., se caracteriza por la presencia de grupos hidróxilos que están unidos a elementos como Si, Al, Ti y Fe. En las fibras naturales estos grupos hidróxilos se encuentran en la superficie de los polímeros como la celulosa [10].

Para lograr una efectiva unión entre el sustrato de origen no polimérico y el adhesivo de origen orgánico es necesario el uso de moléculas bifuncionales, las que actúan como promotores de la adhesión y los silanos son utilizados ampliamente con este propósito. Estos tienen fórmula general $\mathrm{xRSi}(\mathrm{OR} 1)_{3}$ donde el radical $\mathrm{x}$ reacciona con la fase orgánica del polímero del adhesivo y OR1 puede hidrolizarse y condensarse formando enlaces $\mathrm{Si}-\mathrm{O}-\mathrm{Si}$ de siloxano en la superficie del sustrato inorgánico $[11,12]$.

En este trabajo de investigación se realizaron varios procedimientos para mejorar la adherencia entre dos láminas de compuestos de SBR 1502/ harina de madera mediante un adhesivo ecológico de poliuretano. Se realizó modificación a las superficies antes de aplicar el adhesivo, empleando tratamientos como abrasión mecánica, aplicación de APTS, VMTS y halogenación como primers de adhesión.

La madera utilizada para la obtención de la harina es retamo liso, planta considerada como problemática en algunos países de Sudamérica por ser una especie invasora y gran propagadora de llama en incendios forestales. Por lo tanto, este trabajo contribuye a la búsqueda de alternativas para el uso de esta planta y de los residuos derivados de su erradicación.

\section{METODOLOGÍA}

Materiales. Para elaborar las láminas de caucho de estireno butadieno/harina de retamo liso (SBR/ HRL), se empleó fibra natural de retamo liso mercerizada (RL). El tamaño de partícula de la fibra se encuentra en el intervalo 100-800 $\mu \mathrm{m}$, con contenido de humedad de 0,50\%. Como matriz polimérica se utilizó SBR Buna SE 1502H, fabricado por industrias Lanxess ${ }^{\circledR}$. Se empleó como adhesivo poliuretano en base agua (PU), Hidropul $500^{\circledR}$ suministrado por tiendas locales.

También se utilizaron, primer silano de tipo amino APTES:YACA110®, pureza 98,41\%, comercializado por Nanjing Lanya Chem. Co. Ltd. 2., silano tipo vinilo VTMS: Struktol® SCA 972, pureza > $98,6 \%$. Cloro sólido granular al 90\%, adquirido en el mercado local. Resina Tacktificante Unilene ${ }^{\circledR}$ A100 de Basile Quimica ${ }^{\mathrm{TM}}$. Ayuda de proceso: Struktol WB $16^{\circledR}$, de Struktol ${ }^{\circledR}$. Antioxidante: Irganox ${ }^{\circledR} 1076$ manufacturado por BASF ${ }^{\circledR}$. Agente de entrecruzamiento DCP: Perkados ${ }^{\circledR}$ BC FF, pureza 99\%, producido por Akzo Nobel ${ }^{\circledR}$.

Tratamiento de la fibra. La fibra de RL se redujo de tamaño en un molino de martillo NOGUEIRA ${ }^{\circledR}$, modelo DPM junior, velocidad $3.500 \mathrm{rpm}$ y 2,2 $\mathrm{kW}$ de potencia. Posteriormente se mercerizó en una solución de $\mathrm{NaOH}$ al $30 \%$ durante 2 horas, a $23{ }^{\circ} \mathrm{C}[13]$.

Elaboración de las placas de compuesto SBR/ HRL. Para el mezclado de la harina de RL con el SBR 1502 se utilizó un molino de rodillos de cámara abierta, a $120{ }^{\circ} \mathrm{C}$ durante 10 minutos. La mezcla está compuesta por SBR 100 phr, HRL $50 \mathrm{phr}$, resina tacktificante, antioxidante, ayuda de proceso y DCP con $1 \mathrm{phr}$ en cada caso. Las placas se obtienen por "moldeo por compresión" en un molde con dimensiones de $16 \mathrm{~cm}$ x $16 \mathrm{~cm}$ con espesor de 3,45 mm. La temperatura del molde es de $160 \pm 1{ }^{\circ} \mathrm{C}$, presión específica de 5,24 MPa y duración del prensado de 6 minutos.

Diseño experimental. Se realizaron los ocho tratamientos mostrados en la Tabla 1, de los que 
cuatro corresponden a modificación de la superficie con abrasión y otros cuatro a la superficie sin abrasión. De cada tratamiento se efectuaron dos réplicas y aparte del uso de silanos VTMS, APTES y primer clorado, se incluyó un blanco sin tratamiento químico (STQ).

Tabla 1. Descripción de los ensayos.

\begin{tabular}{|c|c|c|c|c|}
\hline \multirow{2}{*}{ Tratamiento } & \multicolumn{4}{|c|}{ Primer } \\
\cline { 2 - 5 } & VTMS & APTES & Clorado & STQ \\
\hline Con abrasión & A-V & A-A & A-H & A-S \\
\hline Sin abrasión & P-V & P-A & P-H & P-S \\
\hline
\end{tabular}

Abrasión de la superficie de la lámina de compuesto SBR/HRL. Para la abrasión de la superficie se utilizó una lija de grano de zirconio de tamaño A100, acoplada a una lijadora de banda marca STANLEY, velocidad libre de $224 \mathrm{rpm}$ con dimensión de la correa $75 \mathrm{~mm}$ x $553 \mathrm{~mm}$.

Aplicación del primers. En cuanto a la preparación y aplicación del primer silano, se hizo disolviendo $6 \mathrm{~g}$ de agente de acoplamiento silano (SCA) en $100 \mathrm{ml}$ de una solución de etanol/agua (80/20, v/v) durante una hora a $25^{\circ} \mathrm{C}$. Se sumergieron las probetas en la solución por cuatro horas, para luego introducirlas en el horno a $55^{\circ} \mathrm{C}$ por 24 horas. Por segunda vez las probetas se introdujeron en el horno a $120^{\circ} \mathrm{C}$ por 2 horas para lograr la incorporación del SCA en el sustrato.

El primer halogenado se preparó disolviendo 2,2 $\mathrm{g}$ de cloro granulado ( $91 \%$ de pureza) en $97,8 \mathrm{~g}$ de acetato de etilo. Pasadas 24 horas de preparada la solución, se esparció una capa sobre la superficie y se secó en un ambiente a $23^{\circ} \mathrm{C}$.

Aplicación del adhesivo. El adhesivo de PU base agua se preparó mezclando $3 \mathrm{~g}$ de toluen-disocianato en $100 \mathrm{~g}$ de PU base agua. Se aplicaron dos capas del adhesivo con un intervalo de 15 minutos. Al evaporarse el agua, las dos placas con el adhesivo se introdujeron en un horno a $120^{\circ} \mathrm{C}$ durante 2 minutos y luego se juntaron utilizando una prensa hidráulica a $30 \mathrm{kgf} / \mathrm{cm}^{2}$ durante 12 segundos.

Medición de resistencia al pelado. En esta prueba se utilizó una máquina universal de ensayos Shimadzu ${ }^{\circledR}$
AGS-X con velocidad de desplazamiento de $50 \mathrm{~mm} /$ min, ambiente de $24^{\circ} \mathrm{C}$ y humedad relativa de $54 \%$. Se utilizaron dos (2) probetas por cada formulación $\mathrm{y}$ se siguieron los lineamientos de procedimiento establecidos en la norma ASTM C794-15A

Caracterización por Espectroscopia FT-IR. Los espectros fueron obtenidos en un equipo NICOLET iS10 ${ }^{\circledR}$. Se mezcló $1 \mathrm{mg}$ de material en polvo con $80 \mathrm{mg}$ de bromuro de potasio para obtener ventanas de $\mathrm{KBr}$ por presión. El rango de longitud de onda se encuentra entre $4.000 \mathrm{~cm}^{-1}$ y $400 \mathrm{~cm}^{-1}$.

\section{Microscopia electrónica de barrido SEM.} La morfología de la superficie modificada del compuesto SBR/HRL se observó en el microscopio FEI QUANTA ${ }^{\circledR} 200$, en condiciones de bajo vacío y aceleración de $30 \mathrm{kV}$. El tratamiento de metalización de la superficie se llevó a cabo en el equipo Balzers ${ }^{\circledR}$ SDC 050 con una película de oro-paladio de $\pm 5 \mathrm{~nm}$ de espesor.

\section{RESULTADOS Y ANÁLISIS}

Resistencia al pelado. Para determinar las propiedades de adhesión se llevaron a cabo ensayos de resistencia al pelado en juntas de SBR/HRL con poliuretano como adhesivo. En la Figura 1, se muestran los valores obtenidos de resistencia al pelado como una función del método de adherencia aplicado. Se omitieron valores menores a $0,50 \mathrm{kN} / \mathrm{m}$ por considerar, de acuerdo con [14], que en estos casos se presenta una baja adherencia entre las probetas.

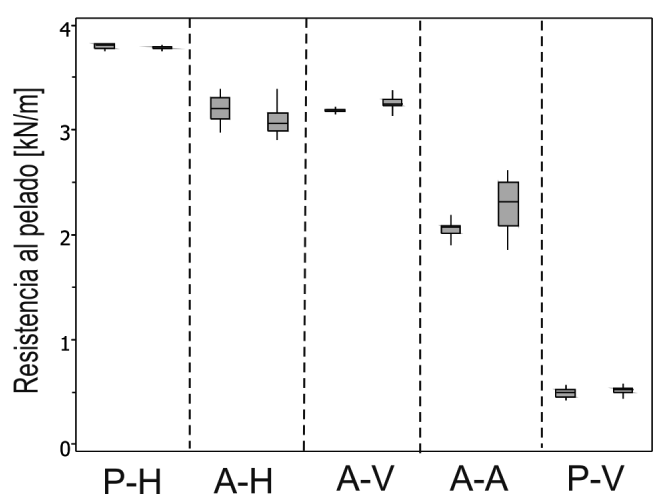

Figura 1. Resistencia al pelado de los diferentes métodos utilizados en la unión de placas SBR/HRL. 
El valor más alto de resistencia al pelado $(3,8 \mathrm{kN} / \mathrm{m})$ corresponde a las probetas $\mathrm{P}-\mathrm{H}$ y posiblemente se debe a que la superficie tratada con cloro se modifica por la formación de enlaces $\mathrm{C}-\mathrm{Cl}$ y $\mathrm{C}=\mathrm{O}$, siendo estos son importantes en la unión química entre el estireno-butadieno y el adhesivo [15]. Además, toda la superficie de contacto del compuesto P-H con el PU está constituida por SBR modificado.

Cuando el primer clorado se aplica en la superficie modificada con abrasión, el valor de resistencia al pelado se reduce hasta $3,21 \mathrm{kN} / \mathrm{m}$ (método A-H). Debido a la presencia de SBR y HRL en la superficie de la probeta tratada con abrasión, el primer clorado no contribuye significativamente entre la unión de PU y la superficie de HRL.

Los valores de resistencia al pelado que presentan los métodos de A-V y P-V (3,23 y $0,48 \mathrm{kN} / \mathrm{m}$ respectivamente), indican como el VTMS es eficaz como primers de adherencia exclusivamente para la unión entre la superficie de HRL y PU.

Se destaca como los valores correspondientes a los métodos A-H $(3.21 \mathrm{kN} / \mathrm{m})$ y A-V $(3.23 \mathrm{kN} / \mathrm{m})$ son similares. En ambos casos la abrasión de la superficie propicia que solo una fracción de la misma se adhiera al PU; en el caso de A-H se da la unión PU y SBR, mientras que en el caso A-V se da entre PU y HRL.

Existe diferencia en los valores de resistencia al pelado de A-V y A-A, correspondiendo el mayor a la utilización de VTMS. Esto sugiere que el aporte del VTMS debido a su estructura química (en particular el grupo vinilo) es más efectivo para el acople entre la fibra y el caucho, que el que puede proporcionar el APTES debido a su grupo amino [16].

Análisis por FT-IR. En la Figura 2, se muestran los espectros de FT-IR del compuesto de SBR con HRL. El compuesto sin tratamiento superficial, Figura 2 (a), presenta las bandas características de los grupos funcionales del SBR. En $2.915 \mathrm{y}$ $2.847 \mathrm{~cm}^{-1}$ se presentan los estiramientos $\mathrm{C}-\mathrm{H}$ (grupos $\mathrm{CH}_{2}$ y $\mathrm{CH}_{3}$ ) propios del butadieno y en $963 \mathrm{~cm}^{-1}$ se aprecian bandas de absorción de butadieno correspondientes al estiramiento de grupos C=C. Los picos en $698 \mathrm{~cm}^{-1}, 760 \mathrm{~cm}^{-1} \mathrm{y}$ $913 \mathrm{~cm}^{-1}$ pertenecen a la absorción del estireno (C-H, fuera del plano de flexión).

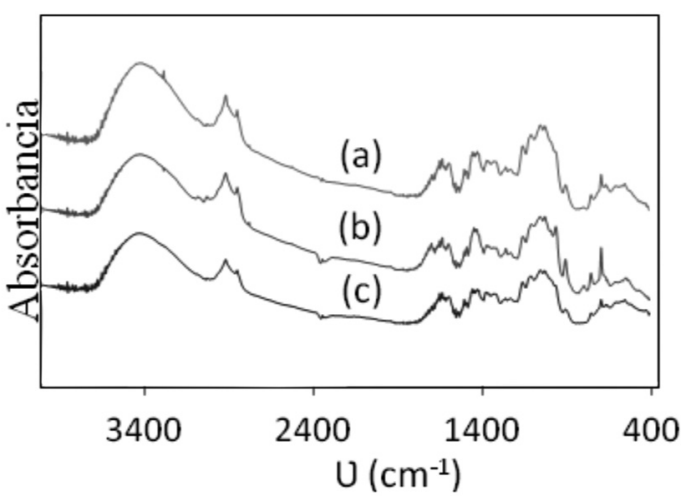

Figura 2. Espectros de FT-IR de superficie de: a) P-S, b) P-H y c) P-H Junta $($ después de despegue).

La Figura 2 (b) muestra el espectro de FT-IR del compuesto en el que se realizó el tratamiento superficial con cloro. Los picos característicos a la cloración se encuentran en las bandas de estiramiento de $\mathrm{C}-\mathrm{Cl}\left(550,786\right.$ y $\left.760 \mathrm{~cm}^{-1}\right)$, como también la oxidación de la superficie del caucho, producto de la cloración, con banda de estiramiento $\mathrm{C}=\mathrm{O}$ en $1.710 \mathrm{~cm}^{-1}$.

En la Figura 2 (c) todos los picos característicos del caucho SBR después del tratamiento superficial con cloro se desvanecen, revelando el espectro del compuesto SBR/HRL que se encuentra debajo de la superficie, lo que sugiere que la capa superior fue desprendida durante el despegue.

La presencia del silano injertado sobre la superficie después del tratamiento también se evaluó mediante FT-IR. El resultado de los espectros de la superficie de P-A, A-A y A-A $\mathrm{A}_{\text {junta }}$ es comparado en la Figura 3. El espectro de P-A (Figura 3 (a)) muestra un aumento en la intensidad de los picos en 2.952 y $2.890 \mathrm{~cm}^{-1}$, relacionados con los grupos $-\mathrm{CH}_{3}$ y $-\mathrm{CH}_{2}$, Aparecen picos entre 900 y $1.400 \mathrm{~cm}^{-1}$, entre 700 y $750 \mathrm{~cm}^{-1}$ y un pico en $1.705 \mathrm{~cm}^{-1}$, regiones donde se observan comúnmente las bandas de estiramiento de los grupos -Si-O-Si- y -Si-O-RUn espectro similar se observa en A-A (Figura 3 (b)) confirmando que el acoplamiento con el SCA se establece no solamente con la fibra, también con el caucho de SBR.

El acoplamiento con la superficie del caucho se puede deber a la combinación del SCA con los 


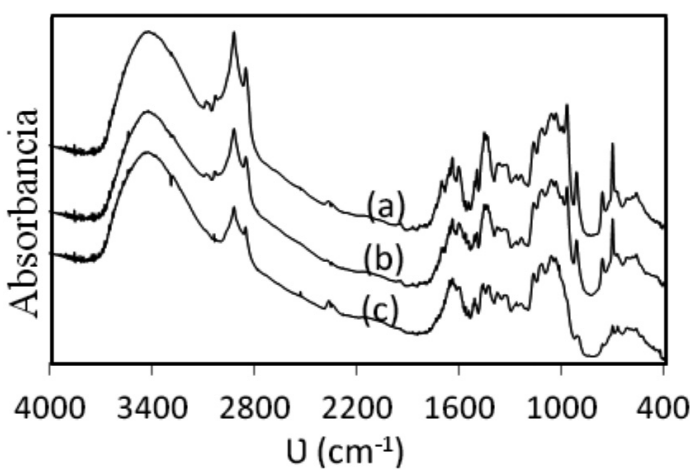

Figura 3. Espectro de FT-IR de superficie de: a) P-A, b) A-A y c) A-A junta (después de despegue).

grupos -OH libres observados en cada uno de los espectros FT-IR en la franja de $3.400 \mathrm{~cm}^{-1}$. Sin embargo, los resultados obtenidos en las pruebas de pelado permiten establecer que estos enlaces son débiles y no contribuyen a la junta entre el PU y el SBR.

En el espectro de A-A $\mathrm{A}_{\text {junta }}$ (Figura 3 (c)), los picos representativos al acoplamiento entre SCA y la superficie de compuesto de caucho y harina de RL con abrasión desaparecen, lo que indicaría que se deben a una capa inferior de la superficie ya que en el momento del despegue, la superficie superior de abr-APTS se quedó adherida a la superficie del PU.

Análisis por SEM. La imagen SEM de la Figura 4 (a) muestra la junta de probetas de SBR/HRL sin abrasión y con modificación superficial por primer de cloro, esto justo cuando se está despegando. En ella se aprecia el estiramiento del adhesivo PU el que está fuertemente adherido a las paredes de las probetas.

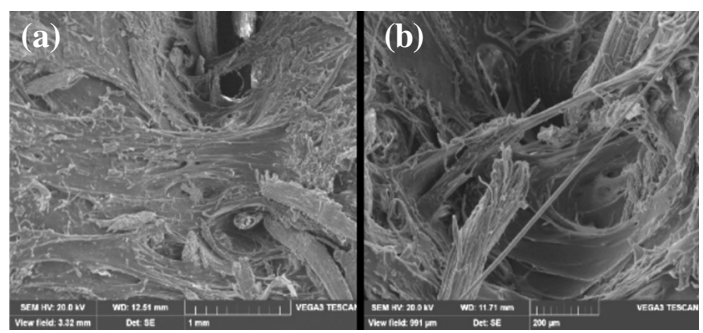

Figura 4. SEM de juntas adhesivas de probetas HRL/SBR. a) P-H, b) A-H.
La Figura 4 (b) ilustra el desprendimiento de las probetas A-H. En este caso existe un alargamiento de filamentos de PU mucho más delgados en comparación con los que se presentan en $\mathrm{P}-\mathrm{H}$ (Figura 4 (a)), debido posiblemente a que no se presenta una adherencia fuerte entre la superficie correspondiente a la fibra de retamo y el PU.

Cuando se analizan las probetas tratadas superficialmente con silanos, en la Figura 5 (a) se aprecia el desprendimiento de las probetas $\mathrm{A}-\mathrm{V}$ con filamentos delgados de PU adheridos a fibra de HRL, situación similar a la que presenta la adhesión de probetas A-H, Figura 4 (b), lo que explica el resultado similar que tienen en resistencia al pelado.

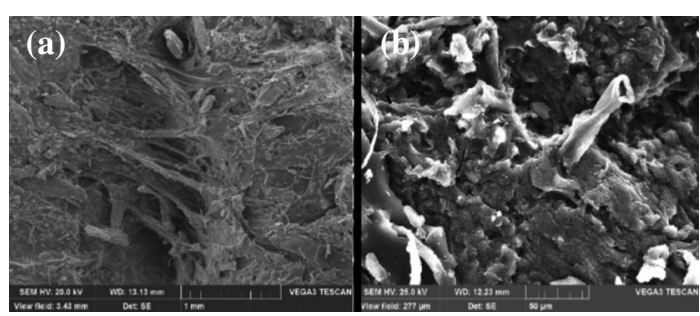

Figura 5. SEM de juntas adhesivas de probetas HRL/SBR. a) A-V b) A-A.

La Figura 5 (b) corresponde al desprendimiento de probetas de A-A, donde se muestra como no existen filamentos producto del estiramiento del PU, siendo coherente con el menor resultado de resistencia al pelado que presenta la prueba correspondiente $\mathrm{a} A-\mathrm{V}$.

\section{CONCLUSIONES}

Se estudiaron seis tratamientos para el estudio de la unión entre láminas de material compuesto SBR/ HRL empleando como adhesivo poliuretano base agua. Los valores más altos de adherencia (medidos mediante resistencia al pelado), se lograron con los siguientes cuatro: tratamiento superficial con cloro (tanto en láminas con y sin abrasión mecánica) y tratamiento superficial con VTMS o APTS en láminas sometidas a abrasión mecánica. Esta unión se corroboró por medio de métodos de caracterización como FT-IR y microfotografías SEM.

Para la superficie modificada con abrasión se establece que el cloro como primers de adhesión es eficaz en la cohesión entre superficie de caucho 
SBR y poliuretano. Asimismo, los silanos VTMS y APTS son eficaces en la cohesión entre superficie harina de retamo liso y poliuretano.

El silano VTMS como primer de adhesión presenta un rendimiento $28,57 \%$ mejor con respecto al APTS. Esto debido posiblemente a que el doble enlace del grupo vinilo del VTMS contribuye mejor que el grupo amino del APTES en la formación de enlaces entre la fibra de retamo liso y el poliuretano.

\section{AGRADECIMIENTOS}

Este estudio es derivado del proyecto de investigación INV-ING-1902, financiado por la Vicerrectoría de Investigaciones de la Universidad Militar Nueva Granada". Vigencia año 2015.

\section{REFERENCIAS}

[1] C.E. Powell and G.W. Beall. "Physical properties of polymer/clay nanocomposites". Current Opinion in Solid State and Materials Science, Vol. $10 \mathrm{~N}^{\circ}$ 2, pp. 73-80. April 2006. ISSN: 1359-0286. DOI: $10.1016 / \mathrm{j}$. cossms.2006.09.001.

[2] S.D. Desai, J.V. Patel, M.R. Patel and V.K. Sinha. "PET waste based polyurethane adhesive for rubber joints". Indian Journal of Chemical Technology. Vol. 12, January 2005, pp. 82-87. ISSN: 0971-457X.

[3] R.P. Kumar, K.C. Manikandan, T. Sabu, S. C. Schit and K. Ramamurthy. "Morphology and melt rheological behaviour of shortsisal-fibre-reinforced SBR composites". Composites Science and Technology. Vol. $60 \mathrm{~N}^{\circ}$ 9, pp. 1737-1751. July 2000. ISSN: 0266-3538/00. DOI: 10.1016/S02 66-3538(00)00057-9.

[4] U. Wisittanawat, S. Thanawan and T. Amornsakchai. "Mechanical properties of highly aligned short pineapple leaf fiber reinforced-Nitrile rubber composite: Effect of fiber content and Bonding Agent". Polymer Testing. Vol. 35, pp. 20-27. May 2014. ISSN: 0142-9418. DOI: 10.1016/j. polymertesting.2014.02.003.

[5] M.J. Owen. "Coupling agent: chemical bonding at interfaces". Dow Corning Corporation. Cap. 9, pp. 404-430. Midland, Ml, USA. ISBN: 978-0-444-51140-9. 2002.
[6] J. Cognard. "Some recent progress in adhesion technology and science". Comptes Rendus Chimie. Vol. $9 \mathrm{~N}^{\mathrm{o}}$ 1, pp. 13-24. January. ISSN: 1631-0748. DOI: 10.1016/j.crci.2004.11.016.

[7] M.D. Romero, M.M. Pastor and J.M. Martin. "Environmental friendly surface treatments of styrene-butadiene-styrene rubber: alternatives to the solvent-based halogenation treatment". International Journal of Adhesion and Adhesives. Vol. 25, $\mathrm{N}^{\mathrm{o}} 1$, pp. 19-29. February 2005. ISSN: 0143-7496. DOI: 10.1016/j.ijadhadh.2004.03.001.

[8] J. Tyczkowski, I. Krawczyk-Kłys, S. Kuberski and P. Makowski. "Chemical nature of adhesion: Plasma modified styrene-butadiene elastomer and polyurethane adhesive joints". European Polymer Journal. Vol. 46, $\mathrm{N}^{\circ} 4$, pp. 767-773. April 2010. ISSN: 0014-3057. DOI: 10.1016/j.eurpolymj.2009.12.019.

[9] M.M. Pastor, J.M. Martin and F.J. Boerio. "Mechanisms of adhesion in surface chlorinated thermoplastic rubber/thermoplastic polyurethane adhesive joints". Rubber Chemistry Technology. Vol. $75 \mathrm{~N}^{\circ} 5$, pp. 825838. November 2002. ISSN: 0035-9475. DOI: $10.5254 / 1.3547686$.

[10] Y. Xie, A.S. Hill, Z. Xiao, H. Militz and C. Mai. "Silane coupling agents used for natural fiber/polymer composites: A review". Composites: Part A. Vol. 41, pp. 806-819. 2010. ISSN: 1359-835X. DOI: $10.1016 / \mathrm{j}$. compositesa.2010.03.005.

[11] L. Fang, L. Chang, W. Guo, Y. Chen. and Z. Wang. "Influence of silane surface modification of veneer on interfacial adhesion of woodplastic plywood". Applied Surface Science. Vol. 288, pp. 682-689. January 2014. ISSN: 0169-4332. DOI:10.1016/j.apsusc.2013.10.098.

[12] T.P. Thao, J.C. Bénézet and A. Bergeret. "Rice and Einkorn wheat husks reinforced poly lactic acid (PLA) biocomposites: Effects of alkaline and silane surface treatments of husks". Industrial Crops and Products. Vol. 58, pp. 111-124. July 2014. ISSN: 0926-6690. DOI: 10.1016/j.indcrop.2014.04.012

[13] O. Buitrago, A. Delgado and W. Aperador. "Surface treatment of straight retamo fiber (Telinne Monspessulana) by silane coupling agents (SCA)". Ciencia e Tecnica Vitivinicola. Vol. $29 \mathrm{~N}^{\circ} 12$, pp. 11-23. 2014. ISSN: 0254-0223. 
[14] M.D. Romero-Sánchez., M.M. Pastor-Blas and J.M. Martin-Martínez. "Adhesion improvement of SBR rubber by treatment with trichloroisocyanuric acid solutions in diferent esters". International Journal of Adhesion and Adhesives. Vol. 21, $\mathrm{N}^{\mathrm{o}} 4$, pp. 325-337. 2001. ISSN: 0143-7496. DOI: 10.1016/S0143-7496(01)00005-7

[15] J. Tyczkowski, I. Krawczyk, B. Wozniak and J.M. Martin-Martínez. "Low-pressure plasma chlorination of styrene-butadiene block copolymer for improved adhesion to polyurethane adhesives". European Polymer Journal. Vol. $45 \mathrm{~N}^{\circ}$ 6, pp. 1826-1835. June, 2009. ISSN: 0014-3057. DOI: 10.1016/j. eurpolymj.2009.02.009

[16] Y. Xie, A.S. Hill, Z. Xiao, H. Militz and C. Mai. "Silane coupling agents used for natural fiber/polymer composites: A review". Composites: Part A. Vol. 41, pp. 806-819. 2010. ISSN: 1359-835X. DOI: $10.1016 / \mathrm{j}$. compositesa.2010.03.005. 